\title{
Fighting muscle weakness in advanced aging by take- home strategies: Safe anti-aging full-body in-bed gym and functional electrical stimulation (FES) for mobility compromised elderly people
}

\author{
Ugo Carraro ${ }^{1}$, Karma Gava ${ }^{2}$, Alfonc Baba ${ }^{1}$, Francesco Piccione ${ }^{1}$ and Andrea Marcante ${ }^{1 *}$ \\ ${ }^{1}$ IRCCS Fondazione San Camillo Hospital, Venice, Italy \\ ${ }^{2}$ Documentary filmaker, Padova, Italy
}

\begin{abstract}
All permanent or progressive muscle contractile impairments (including advanced aging-related muscle strength decline) need permanent management. A homebased physical exercise approach may be helpful. Development of implantable devices for muscle stimulation and of electroceuticals, as effective as pace-makers for cardiac arrhythmias or cochlear implants for hearing loss are still in the future and education of hospitalized patients to take-home physical exercise managements is an effective low cost alternative. Frail elderly people due to advanced age or associated diseases are often hospitalized for long periods of time. In the hospital, their already modest amount of daily physical activity is further reduced, contributing to limit their independence to enable them to leave their beds. Inspired by the proven capability to recover skeletal muscle contractility and strength by home-based Functional Electrical Stimulation even in the worse cases of neuromuscular traumatic injuries, but, guided by common sense, we suggest a short (15-20 minutes) daily routine of twelve easy-to-perform physical exercises that may be performed in bed (Full-body In-Bed Gym). If borderline sedentary persons challenge themselves, within a few days of inpatient care, the Full-body In-Bed Gym may increase muscle strength, fatigue resistance and independence in daily life activities. In surgical units, this will permit standing of patients soon after the surgery, a mandatory measure to prevent risk of thromboembolism. Full-body In-Bed Gym helps also to mitigate the bad mood that many times accompanies mobility limitations, strengthening patients' confidence in recovering partial or total independence. Continued regularly, Full-body In-Bed Gym may help to maintain the independence of frail older people and to reduce the risks of the possible serious consequences of accidental falls.
\end{abstract}

Take home message: For a long-term resting muscle the most important event is the first active muscle contraction. To transform you from a sedentary, overweight, depressed person into a more active senior, there is no need of personal trainers or devices. Read here, please, watch the video and secure a better life.

\section{Introduction}

All permanent and/or progressive muscle contractile impairments (including aging-related muscle strength decline) need permanent management. Immobility in intensive care units is associated with neuromuscular weakness, post-intensive care syndrome, functional limitations, and high hospital costs. Early mobility-based rehabilitation in intensive care units is feasible and safe [1]. Unfortunately, much less attention is paid to regular hospitalized patients, to those from long term care units. Frail elderly due to advanced age or associated diseases are often hospitalized for long periods of time. In the hospital, long term care unit, their already modest amount of daily physical activity is reduced, contributing to limit their independence and confine them for the most part to the bed. Education of hospitalized patients to take-home physical exercise programs is an effective low cost option. Inspired by the proven capability to recover skeletal muscle contractility and strength by home-based Functional Electrical Stimulation (h-bFES) even in the worse cases of neuromuscular traumatic injuries [2-4], and guided by common sense, we suggest a short (10-20 minutes) daily sequence of fifteen easy-to-perform safe physical exercises that may be performed in bed (Full-body In-Bed Gym). If borderline sedentary people persons challenge themselves, within the Full-body In-Bed Gym program may increase muscle strength, fatigue resistance and independence in daily life activities. In surgical units, this will permit standing of patients sooner after surgery, a mandatory measure to prevent risk of thromboembolism. The Fullbody In-Bed Gym program helps also to mitigate the bad mood that accompanies mobility limitations, strengthening confidence of patients in recovering partial or total independence $[5,6]$. Continued regularly, Full-body In-Bed Gym may help to maintain the independence of frail older people and to reduce the risks of the possible serious consequences of accidental falls.

It is never too early, it is never too late to start Anti-Aging Fullbody In-Bed Gym!

Active persons, i.e., those able to make 25 consecutive push-ups,

Correspondence to: Dr. Andrea Marcante, IRCCS Fondazione Ospedale San Camillo, Via Alberoni 70, I-30126 Venezia-Lido, Italy; E-mail: Andreamarcante@ospedale.sancamillo.net

Key words: full-body In-Bed Gym, FES, muscle, atrophy, weakness, aging, home strategies

Received: November 07, 2016; Accepted: November 28, 2016; Published: November 30, 2016 
Carraro U (2016) Fighting muscle weakness in advanced aging by take-home strategies: Safe anti-aging full-body in-bed gym and functional electrical stimulation (FES) for mobility compromised elderly people

do not need the exercises shown in Figure 1. All other persons may start with 5 repetitions of each suggested exercise of the In-Bed Gym Program:

(Figure 1. A, B, Flexion and extension of ankles; C, Cycling movements; D, E, Arms up and down; F, Deep breathing; G and H, Forward bending; I, To raise your pelvis; J, K, L, Neck torsions; M, Lift the body; N, O, Lift the legs; P, stand up from bed; Q, Get up on his toes; R, Push up (Active person). After every successive week, they may add groups of 5 additional exercises up to 30 . The routine may last from 10 (at the beginning of the muscles' training) to 30 minutes, when 30 repetitions of each exercise are reached.

Figure 1 shows the series of safe and easy exercises that elderly persons could perform resting in bed. Figure 1, A and B suggest to start every morning the Full-body In-Bed Routine with flexions and extensions of ankles; Figure 1, C and D demonstrate that leg and abdomen muscle could be activated by cycling movements; Figure 1, $\mathrm{E}$ and $\mathrm{F}$ show arm up and down exercise. Notice, please, that hand muscles are also activated; Figure 1, G show how deep breathing could be performed; Figure 1, H and I show forward bending exercise; Figure $1, \mathrm{~J}$ and $\mathrm{K}$ suggest how to raise your pelvis to activate back muscles, glutei and posterior leg muscles; Figure 1, L to $\mathrm{O}$ demonstrate neck

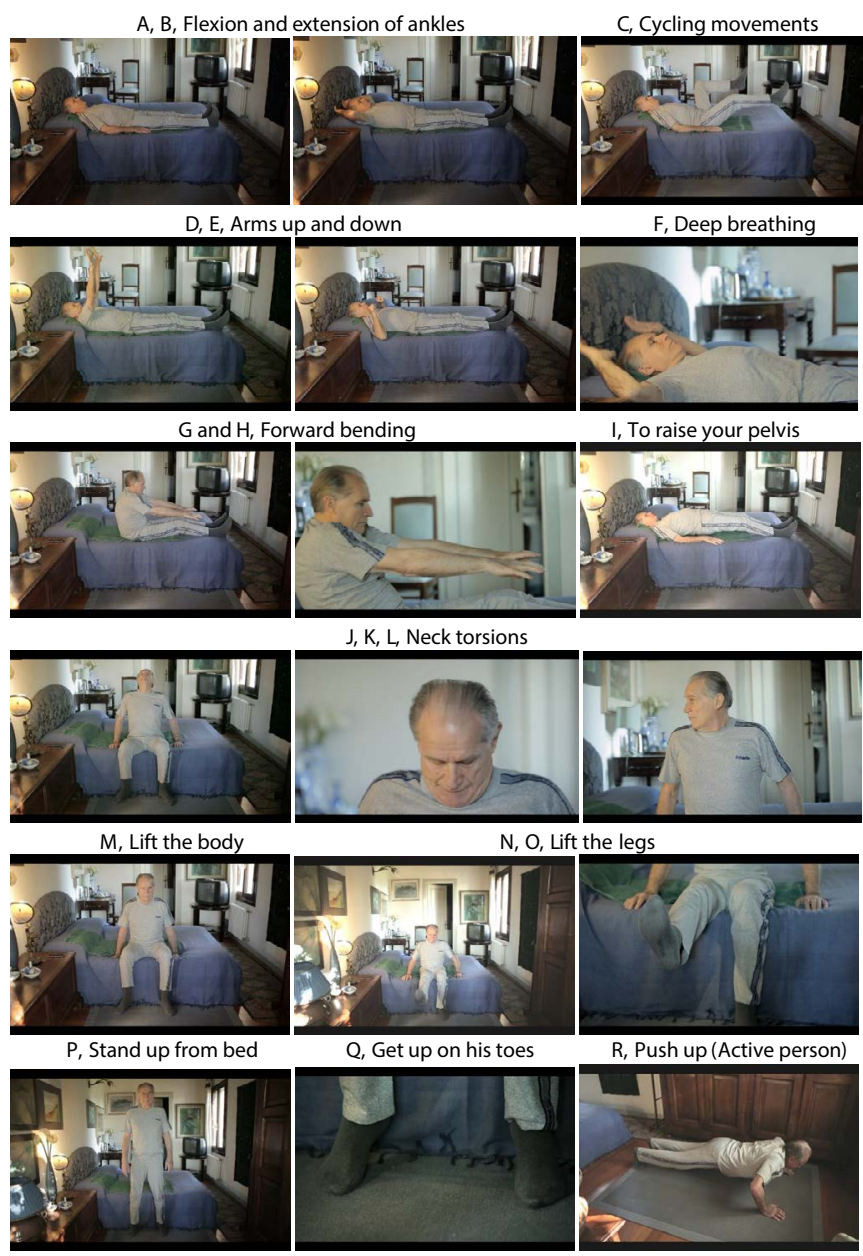

Figure 1. Full-body In-Bed Gym for elderly or mobility impaired persons.

("A, B, Flexion and extension of ankles; C, Cycling movements; D, E, Arms up and down; F, Deep breathing; G and H, Forward bending; I, To raise your pelvis; J, K, L, Neck torsions; M, Lift the body; N, O, Lift the legs; P, Stand up from bed; Q, Get up on his toes; $\mathrm{R}$, Push up (Active person)") torsions; Figure 1, P suggest to lift the upper part of the body in sitting position to exercise arm muscles; Figure $1, \mathrm{P}$ and $\mathrm{Q}$, demonstrate how to lift the legs in sitting position to strength quadriceps muscles; Figure $1, S$ and $\mathrm{T}$ show standing up and get up on his toes, respectively. Further, a video may be downloaded from the link: http://www.bio. unipd.it/bam/video/InterviewCarraro-tutorial.mp4

If elderly persons can't, or are reluctant to, perform physical exercises, functional electrical stimulation may mimic them and be almost equally useful in terms of maintaining skeletal muscle strength and endurance [2-4].

Aging is an unavoidable process, if lethal diseases do not prematurely end survival. Strong evidence of this concept has been recently provided by Gava et al., 2013 [7], describing the power decay of the series of World Records obtained by Master Athletes (from 30 to 100 years).

Skeletal muscle strength with age, indeed, continues to decline, almost linearly, to zero at the age of 110 years. Recently, we have discovered evidence that denervation of muscle fibers contributes to atrophy and slowness of aged muscles by comparing muscles of lifelong active seniors to those of sedentary elderly people [8]. We found that the sportsmen have more muscle mass and slow fiber groupings, providing in this way evidence that physical activity maintains slow motoneurons, which re-innervate muscle fibers which were previously denervated [9-11]. On the other hand, a premature muscle atrophy and degeneration occurs with irreversible and complete conus and cauda equine syndrome consequent to a lumbar/sacral spinal cord injury. Under this condition a complete loss of muscle fibers within five-eight years is expected [12-14].

The impact of muscle denervation on aging skeletal muscle fibers is a relatively new topic $[15,16]$. This is related in part to the difficulties in using molecular approaches to determine whether motor neurons release chemical neurotrophic agents to the muscle fibers of the motor units. Such a mechanism contributes to the development and maintenance of the neuromuscular junctions. However, if and which chemical trophic factors influence the synchronized expression of the hundreds of nuclei belonging to a single muscle fiber remains unknown. The synchronized spread of muscle action potential seems to be, on the contrary, a more rational mechanism [17]. Despite this opinion, the conclusions of recent reviews are that functional electrical stimulation (FES) is poorly used and ineffective in rehabilitation of elderly patients $[14,15,18]$. Aging skeletal muscle presents both a loss of muscle mass and a conspicuous reduction in myofiber plasticity [15,18-20], by alterations in muscle-specific transcriptional mechanisms. During the aging process, protein synthetic rates decrease and an increase in protein degradation follows $[4,21,22]$.

Ineffective damage repair is a contributory cause of functional impairment during aging [23-25], promoting the detrimental replacement of functional contractile muscle with fibrous connective tissue [22]. Volitional physical exercise can reverse these processes. Interestingly, it has been shown that both acute and prolonged resistance exercise stimulates the proliferation of satellite cells [27]. This fact may be explained by the increased levels of myostatin, a negative regulator of muscle mass. Furthermore, it has been reported an increase in autophagy in the muscle of athletic people, suggesting that exercise may activate important systems that detoxify muscle cells [28]. Other evidence suggests that training and regular exercise increase muscle strength and attenuate sarcopenia by modulating expression of autophagy, myokines (IL-6) and IGF-1 $[29,30]$. 
Carraro U (2016) Fighting muscle weakness in advanced aging by take-home strategies: Safe anti-aging full-body in-bed gym and functional electrical stimulation (FES) for mobility compromised elderly people

Unfortunately, elderly people may be unable or reluctant to adequately participate in physical exercise. FES could be an alternative. Stimulators for neuromuscular electrical stimulation (ES) are especially suited for elderly people requirements [31]. As detailed in Kern et al., 2014 [4], older persons may be exposed to regular neuromuscular ES training. These constant voltage stimulation devices can be safely applied during home use. Starting two times a week for the first 3 weeks and then switching to three times a week for the next 6 weeks, for a total amount of 24 training sessions $(3 \times 10$ minutes for each session) ES is safe and effective. After detailed instructions, ES training may be performed by the subjects themselves at home, using a two-channel custom-built battery-powered stimulator [26] The subjects apply two conductive rubber electrodes $\left(9 \times 14 \mathrm{~cm} ; 126 \mathrm{~cm}^{2}\right)$ which are attached to the skin by wet sponge on the anterior thigh on both sides (left/right). The electrode pairs for left and right thigh are connected to the two channels of the stimulator. This allows independent activation of the left and right thigh muscles, which are alternatively stimulated. Each repetition (muscle contraction) was evoked by a $3.5 \mathrm{~s}$ train $(60 \mathrm{~Hz})$ of electrical pulses (rectangular, biphasic, width $0.6 \mathrm{~ms}$ ). Consecutive contractions of the same thigh are separated by $4.5 \mathrm{~s}$ off intervals. The subjects ought to be instructed to increase the stimulation intensity until their maximal tolerance is reached. Using this approach a full knee extension is achieved in all subjects. Nevertheless, a mean stimulation current of $128+/-16 \mathrm{~mA}$ and voltage of $39+/-14 \mathrm{~V}$ may be well tolerated. The outcome is a significant increase in muscle strength, associated with an increase of fast muscle fibers, which are the first to respond to ES and are well related to the power of skeletal muscle $[4,20]$. We observed in our group of elderly persons that ES increases expression of IGF1, the markers of satellite cell proliferation and extracellular matrix remodeling, downregulating the expression of proteases $[4,20]$. There is also a collagen remodeling during both volitional physical exercise and ES. Three different forms of collagen are up-regulated in electrically stimulated muscle [14,15], but the increase in collagen does not likely stimulate fibrosis, as is shown by both morphological evidence and the increase in expression of miR29 [32]. Functional tests of trained subjects showed that there are ES induced improvements of leg muscle strength and mobility [33,34]. ES significantly increased the size (diameter) of fast type muscle fibers, and the number of Pax7- and NCAM-positive satellite cells. Moreover, no signs of muscle damage and/or inflammation were observed in analyzed muscle biopsies $[4,20]$. Altogether, these results demonstrate that physical exercise, either voluntary or induced by ES, improves the functional performance of aging muscles. Of course, physical training can't stop the aging process, but ES is a safe home-based method that can counteract atrophy of fast twitch muscle fibers.

Age-related muscle power strength is partially attributable to a loss of innervation and it can be delayed by a life-long high-level activity [3-5]. Diseases involving permanent denervation show a similar, but premature, aging process and much more severe muscle deterioration. Despite doubts and criticisms of related literature [35,36], we have shown that, with appropriate protocols, h-bFES can even inhibit degeneration of denervated muscle and even reverse it $[2,37,38]$. Therefore, use of FES should be extended from critical care units to rehabilitation centers, nursing facilities and at home of the elderly population [39-42].

\section{References}

1. Hopkins RO, Mitchell L, Thomsen GE, Schafer M, Link M, et al. (2016) Implementing a Mobility Program to Minimize Post-Intensive Care Syndrome. AACN Adv Crit Care 27: 187-203.[Crossref]
2. Kern H, Carraro U, Adami N, Biral D, Hofer C, et al. (2010) Home-based functional electrical stimulation rescues permanently denervated muscles in paraplegic patients with complete lower motor neuron lesion. Neurorehabil Neural Repair 24: 709-721. [Crossref]

3. Carraro U, Kern H, Gava P, Hofer C, Loefler S, et al. (2015) Biology of Muscle Atrophy and of its Recovery by FES in Aging and Mobility Impairments: Roots and By-Products. Eur J Transl Myol 25: 221-230.[Crossref]

4. Kern H, Barberi L, Löfler S, Sbardella S, Burggraf S, et al.(2014) Electrical stimulation counteracts muscle decline in seniors. Front Aging Neurosci 6: 189. [Crossref]

5. Carneiro LS, Fonseca AM, Serrão P, Mota MP, Vasconcelos-Raposo J, et al. (2016) Impact of physical exercise on catechol-O-methyltransferase activity in depressive patients: A preliminary communication. J Affect Disord 193: 117-122. [Crossref]

6. Börjesson M, Onerup A, Lundqvist S, Dahlöf B (2016) Physical activity and exercise lower blood pressure in individuals with hypertension: narrative review of $27 \mathrm{RCTs} . \mathrm{Br}$ J Sports Med 50: 356-361.[Crossref]

7. Gava P, Kern H, Carraro U (2015) Age-associated power decline from running, jumping, and throwing male masters world records. Exp Aging Res 41: 115-135.[Crossref]

8. McKinnon NB, Connelly DM, Rice CL, Hunter SW, Doherty TJ (2016) Neuromuscular contributions to the age-related reduction in muscle power: Mechanisms and potential role of high velocity power training. Ageing Res Rev S1568-1637: 30164-30167. [Crossref]

9. Mosole S, Rossini K, Kern H, et al. (2013) Significant increase of vastus lateralis reinnervation in 70-year sportsmen with a lifelong history of high-level exercise. Eur $J$ Transl Myol - Basic Appl Myol 23: 117-122.

10. Mosole S, Carraro U, Kern H, Loefler S, Fruhmann H, et al. (2014) Long-term highlevel exercise promotes muscle reinnervation with age. J Neuropathol Exp Neurol 73 284-294.[Crossref]

11. Mosole S, Carraro U, Kern H, Loefler S, Zampieri S (2016) Use it or lose it: tonic activity of slow motoneurons promotes their survival and preferentially increases slow fiber-type groupings in muscles of old lifelong recreational sportsmen. Eur J Transl Myol 26: 339-346.

12. Kern H, Carraro U, Adami N, Hofer C, Loefler S, et al. (2010) One year of home-base Functional Electrical Stimulation (FES) in complete lower motor neuron paraplegia: Recovery of tetanic contractility drives the structural improvements of denervated muscle. Neurol Res 32: 5-12.[Crossref]

13. Kern H, Boncompagni S, Rossini K, Mayr W, Fanò G, et al. (2004) Long-term denervation in humans causes degeneration of both contractile and excitationcontraction coupling apparatus that can be reversed by functional electrical stimulation (FES). A role for myofiber regeneration? J Neuropathol Exp Neurol 63: 919-931. [Crossref]

14. Hepple RT, Rice CL (2016) Innervation and neuromuscular control in ageing skeletal muscle. J Physiol 594:1965-7198. [Crossref]

15. Carraro U, Kern H, Gava P, Hofer C, Loefler S, et al. (2016) Recovery from muscle weakness by exercise and FES: lessons from Masters, active or sedentary seniors and SCI patients. Aging Clin Exp Res.[Crossref]

16. Carraro U, Kern H (2016) Severely Atrophic Human Muscle Fibers with Nuclear Misplacement Survive Many Years of Permanent Denervation. Eur J Transl Myol26:5894. [Crossref]

17. Lomo T (2014) The Response of Denervated Muscle to Long-Term Stimulation (1985, Revisited here in 2014). Eur J Transl Myol 24: 3294.[Crossref]

18. Mitchell WK, Williams J, Atherton P, Larvin M, Lund J, et al. (2012) Sarcopenia, dynapenia, and the impact of advancing age on human skeletal muscle size and strength; a quantitative review. Front Physiol 3: 260.[Crossref]

19. Zampieri S, Mosole S, Löfler S, Fruhmann H, Burggraf S, et al. (2015) Physical exercise in Aging: Nine weeks of leg press or electrical stimulation training in 70 years old sedentary elderly people. Eur J Transl Myol 25: 237-242.[Crossref]

20. Zampieri S, Pietrangelo L, Loefler S, Fruhmann H, Vogelauer M, et al. (2015) Lifelong physical exercise delays age-associated skeletal muscle decline. J Gerontol A Biol Sci Med Sci 70: 163-173.[Crossref]

21. Carnio S, LoVerso F, Baraibar MA, Longa E, Khan MM, et al. (2014) Autophagy impairment in muscle induces neuromuscular junction degeneration and precocious aging. Cell Rep 8: 1509-1521. [Crossref]

22. Barber L, Scicchitano BM, Musaro A (2015) Molecular and Cellular Mechanisms of Muscle Aging and Sarcopenia and Effects of Electrical Stimulation in Seniors. Eur J Transl Myol 25: 231-236.[Crossref] 
Carraro U (2016) Fighting muscle weakness in advanced aging by take-home strategies: Safe anti-aging full-body in-bed gym and functional electrical stimulation (FES) for mobility compromised elderly people

23. Scicchitano BM, Rizzuto E, Musarò A (2009) Counteracting muscle wasting in aging and neuromuscular diseases: the critical role of IGF-1. Aging (Albany NY) 1: 451-457. [Crossref]

24. Vinciguerra M, Musaro A, Rosenthal N (2010) Regulation of muscle atrophy in aging and disease. Adv Exp Med Biol 694: 211-233.[Crossref]

25. Carosio S, Berardinelli MG, Aucello M, Musarò A (2011) Impact of ageing on muscle cell regeneration. Ageing Res Rev 10: 35-42.[Crossref]

26. Barberi L, Scicchitano BM, De Rossi M, Bigot A, Duguez S, et al. (2013) Age-dependent alteration in muscle regeneration: the critical role of tissue niche. Biogerontology 14: 273-292.[Crossref]

27. Musarò A (2014) The Basis of Muscle Regeneration. Advances in Biology, 2014: 1-16.

28. Sandri M, Barberi L, Bijlsma AY, Blaauw B, Dyar KA, et al. (2013) Signalling pathways regulating muscle mass in ageing skeletal muscle: the role of the IGF1-AktmTOR-FoxO pathway. Biogerontology 14: 303-323.[Crossref]

29. Adamo ML, Farrar RP (2006) Resistance training, and IGF involvement in the maintenance of muscle mass during the aging process. Ageing Res Rev 5: 310-331. [Crossref]

30. Pelosi L, Berardinelli MG, De Pasquale L, Nicoletti C, D’Amico A, et al. (2015) Functional and Morphological Improvement of Dystrophic Muscle by Interleukin 6 Receptor Blockade. EBioMedicine 2: 285-293.[Crossref]

31. Krenn M, Haller M, Bijak M, Unger E, Hofer C, et al. (2011) Safe neuromuscular electrical stimulator designed for the elderly. Artif Organs 35: 253-256.[Crossref]

32. He Y, Huang C, Lin X, Li J (2013) MicroRNA-29 family, a crucial therapeutic target for fibrosis diseases. Biochimie 95: 1355-1359.[Crossref]

33. Cvecka J, Tirpakova V, Sedliak M, Kern H, Mayr W, et al. (2015) Physical Activity in Elderly. Eur J Transl Myol 25: 249-252.[Crossref]
34. Sarabon N, Löfler S, Hosszu G, Hofer C (2015) Mobility Test Protocols for the Elderly: A Methodological Note. Eur J Transl Myol 25: 253-256.[Crossref]

35. Hughes AM, Burridge JH, Demain SH, et al. (2014) Translation of evidence-based assistive technologies into stroke rehabilitation: users' perceptions of the barriers and opportunities. BMC Health Serv Res 14: 124. [Crossref]

36. Bersch I, Tesini S, Bersch U, Frotzler A (2015) Functional electrical stimulation in spinal cord injury: clinical evidence versus daily practice. Artif Organs 39: 849-854. [Crossref]

37. Kern H, Hofer C, Moedlin M, Forstner C, Raschka-Hoger D,et al. (2002) Denervated muscle in humans: limitations and problems of currently used functional electrical stimulation training protocols. Artif Organs 26: 216-218. [Crossref]

38. Kern H, Carraro U (2014) Home-based Functional Electrical Stimulation (h-b FES) for long-term denervated human muscle: History, basics, results and perspectives of the Vienna Rehabilitation Strategy. Eur J Transl Myol 24: 3296. [Crossref]

39. Willand MP (2015) Electrical Stimulation Enhances Reinnervation After Nerve Injury. Eur J Transl Myol 25: 243-248.[Crossref]

40. Catapano J, Willand MP, Zhang JJ, Scholl D, Gordon T, et al. (2016) Retrograde labeling of regenerating motor and sensory neurons using silicone caps. $J$ Neurosci Methods 259:122-128. [Crossref]

41. Willand MP, Nguyen MA, Borschel GH, Gordon T (2016) Electrical Stimulation to Promote Peripheral Nerve Regeneration. Neurorehabil Neural Repair 30: 490-496. [Crossref]

42. Willand MP, Rosa E, Michalski B, Zhang JJ, Gordon T, et al. (2016) Electrical muscle stimulation elevates intramuscular BDNF and GDNF mRNA following peripheral nerve injury and repair in rats. Neuroscience 334: 93-104. [Crossref]

Copyright: $\odot 2016$ Carraro U. This is an open-access article distributed under the terms of the Creative Commons Attribution License, which permits unrestricted use, distribution, and reproduction in any medium, provided the original author and source are credited. 